\title{
Bodenbesiedelnde Spinnen (Arachnida, Araneae) eines Kiefernforstes bei Stücken in Brandenburg, Deutschland
}

\author{
Volker NICOLAI \& Andreas HERRMANN
}

\begin{abstract}
Ground living spiders (Arachnida, Araneae) of a pine forest near Stücken in Brandenburg, Germany.

Ground inhabiting spiders were investigated during the vegetation periods of 1996,1998 and 2000 in four pine forests of Brandenburg, Germany. 1995 three of them were managed in different ways and one area served as a control. In total 105 different species were found and the lowest number of species was found on the control area. Due to the management the pine forests got opened and xerophilous species could settle the areas. In total a heterogeneous species community was found on each area. From year to year high rates of species turnover were measured even for the dominant species $(>5 \%$ of all collected individuals per area and year). Some endangered species in Brandenburg were collected as well.
\end{abstract}

Key words: Ground living spider fauna, pine forests, Brandenburg, communities, alteration of species assemblages.

\section{EINLEITUNG}

Die heutige Baumartenverteilung in Deutschland entspricht aus verschiedenen Gründen nicht einer naturnahen Baumartenzusammensetzung (ELLENBERG 1996, PETERKEN 1996, POTT 1997, REMMERT 1998). Der Anteil von Kiefern und von Fichten wurde in den letzten Jahrhunderten stark erhöht (SCHERZINGER 1996). So beträgt der gesamte Flächenanteil der Nadelbäume in Westdeutschland $69 \%$, in Ostdeutschland $79 \%$, und hier bestehen $54 \%$ der Gesamtwaldfläche aus Kiefern (ELLENBERG 1996). Auf vergleichsweise großen Flächen werden solche Kiefernforste langfristig in Laubmischwälder überführt (AMMER \& KÜBLER 1998). Wie eine solche Überführung erreicht werden kann und welche Auswirkungen die durchgeführten Maßnahmen auf die bodenbesiedelnde Spinnenfauna haben kann, war eine Ausgangsfrage dieser Studie. Dazu wurden 1995 in für Brandenburg derzeit typischen Kiefernreinbeständen 
südlich von Berlin drei Varianten von Umbaumaßnahmen angelegt und mit einer Kontrolle verglichen. Es wurden möglichst typische Versuchsflächen ausgewählt, um eine Übertragbarkeit auf andere Kiefernreinbestände zu ermöglichen. Umbaumaßnahmen jeglicher Art sollten zuerst Veränderungen in der Bodenfauna zeigen. Mit standardisierten Methoden wurde daher die Bodenfauna während der Vegetationsperioden 1996, 1998 und 2000 untersucht. Hier soll über die bodenbesiedelnden Spinnengemeinschaften und ihre Veränderungen in den Kiefernforsten berichtet werden.

\section{UNTERSUCHUNGSGEBIET}

Die Untersuchungen wurden in Brandenburg (Landkreis Potsdam Mittelmark, nahe Stücken: $52^{\circ} 18^{\circ} \mathrm{N} 13^{\circ} 2^{\circ} \mathrm{O}$, TK 25) in der Nuthe-NieplitzNiederung etwa $40 \mathrm{~km}$ südwestlich von Berlin durchgeführt. Dieses Fördergebiet von gesamtstaatlich repräsentativer Bedeutung besitzt eine Kerngebietsgröße von etwa 5000 ha und eine Projektgebietsgröße von etwa 110.000 ha (BUNDESAMT FÜR NATURSCHUTZ 1999). Hier werden u.a. waldbauliche Veränderungen der Kiefernforste in Kiefern-Laubmischwälder auf etwa $25 \%$ der Gebietsfläche durchgeführt (BUNDESAMT FÜRNATUR-SCHUTZ 1999). Weitere Angaben zum Untersuchungsgebiet finden sich bei SEELEMANN etal.(1996) und NICOLAI \& MACHANDER (2000).

\section{UNTERSUCHUNGSFLÄCHEN}

Drei etwa gleich große (4ha), aneinander angrenzende, gebietsrepräsentative und gleichaltrige (ca. 100 Jahre) Kiefernforste wurden ausgewählt und im Herbst 1995 unterschiedlich behandelt. Untersuchungsfläche 1: Durchforstung, Stammentnahme (10\%), Räumung und Aufschichtung des Kronenholzes, Reihenbepflanzung (Abstand der Pflanzreihen ca. 3 - $5 \mathrm{~m}$ ) mit Eichen, Hainbuchen und Ahorn. Zusätzlich erfolgte eine Einzäunung der Versuchsfläche mit einem $2 \mathrm{~m}$ hohen Zaun. Untersuchungsfläche 2: Durchforstung mit gleicher Intensität und Stammentnahme, das Kronenholz verblieb am Ort, es erfolgten keine Pflanzungen und auch keine Einzäunung. Untersuchungsfläche 3: Kontrollfläche ohne Maßnahmen. Im Herbst 1997 
wurde eine zusätzliche Untersuchungsfläche eingerichtet, die ebenfalls unbehandelt blieb, aber mit einem $2 \mathrm{~m}$ hohen Zaun umgeben wurde und als Untersuchungsfläche 0 bezeichnet wurde.

\section{MATERIAL UND METHODEN}

Auf den Untersuchungsflächen wurden die bodenbesiedelnden Spinnen mit je 10 Bodenfallen pro Untersuchungsfläche in den Jahren 1996, 1998 und 2000 erfasst. Als Fixiermittel diente Ethylenglykol (70 \%), der Durchmesser der Fallen betrug $6,5 \mathrm{~cm}$ und der Mindestabstand zwischen den Fallen betrug $>2 \mathrm{~m}$. Von April bis Oktober waren die Fallen monatlich für 10 - 14-tägige Fangperioden exponiert. Im Labor wurden die fixierten Tiere in 70 \% Ethanol überführt, gezählt und nach WIEHLE $(1956,1960)$, HEIMER \& NENTWIG (1991) sowie ROBERTS (1995) bestimmt. Die Nomenklatur folgt PLATNICK (1997). Statistische Auswertungen erfolgten mit SPSS Version 10.07.

\section{ERGEBNISSE}

1996 wurden auf allen Untersuchungsflächen 792 Individuen mit insgesamt 69 Arten nachgewiesen (Tab. 1). Auf den Untersuchungsflächen 1 und 2 konnten 45 bzw. 42 Arten und auf der Kontrollfläche 3 konnten 29 Arten gefunden werden (Tab. 1). Die Aktivitätsdichten (gefangene Individuen pro Falle pro Tag $=\mathrm{n} / \mathrm{FxT}$ ) wiesen auf der Untersuchungsfläche 1 mit 0,532 Individuen pro Falle pro Tag höhere Werte auf als auf den Untersuchungsflächen $2(0,323)$ und $3(0,251)$. Bemerkenswert ist der Nachweis von Araeoncus crassiceps von der Kontrollfläche 3. Die Art wird in der Katergorie 3 der Roten Liste der Spinnentiere von Berlin und Brandenburg geführt (PLATEN et al. 1999). Sie bevorzugt feuchte Lebensräume wie Moore und ist zuletzt 1978 bei Beeskow (Kreis Oder-Spree) gefunden worden (PLATEN et al. 1999). Hervorzuheben ist auch der Nachweis von Scotina palliardi auf der Untersuchungsfläche 1. Die Art wird in Kategorie 2 der Roten Liste der Spinnentiere von Berlin und Brandenburg geführt (PLATEN et al. 1999). Sie bevorzugt trockene Lebensräume wie Trockenrasen und wurde zuletzt 1996 aus dem unteren Odertal gemeldet (PLATEN et al. 1999). 
Tab. 1: Aktivitätsdichten (Individuenanzahlen pro Falle pro Tag: n/FxT) (oberer Wert) und prozentualer Anteil pro Untersuchungsfläche pro Jahr (unterer Wert) der Spinnen (Arachnida: Araneae) auf den Untersuchungsflächen (0-3) in den Jahren 1996, 1998 und 2000, Summen, Artenanzahlen pro Untersuchungsfläche und turnover.

Tab. 1: The numbers of spider species (Arachnida: Araneae) collected per day per trap (first value) and in percentage per study area and year (second value) on the study areas (0-3) during the years 1996, 1998 and 2000, sum, numbers of species per study area and turnover per year.

\begin{tabular}{|c|c|c|c|c|c|c|c|c|c|c|c|}
\hline Fläche & 0 & 0 & 1 & 1 & 1 & 2 & 2 & 2 & 3 & 3 & 3 \\
\hline Jahr & 1998 & 2000 & 1996 & 1998 & 2000 & 1996 & 1998 & 2000 & 1996 & 1998 & 2000 \\
\hline $\begin{array}{l}\text { 1. Abacoproeces saltuum } \\
\text { (L. KOCH) }\end{array}$ & $\begin{array}{c}0,002 \\
1,0\end{array}$ & & & $\begin{array}{c}0,001 \\
0,2\end{array}$ & $\begin{array}{c}0,001 \\
0,6\end{array}$ & $\begin{array}{c}0,012 \\
3,9\end{array}$ & $\begin{array}{c}0,011 \\
1,7\end{array}$ & $\begin{array}{c}0,007 \\
1,4\end{array}$ & $\begin{array}{c}0,004 \\
1.6\end{array}$ & $\begin{array}{c}0,001 \\
0,7\end{array}$ & $\begin{array}{c}0,001 \\
0,7\end{array}$ \\
\hline $\begin{array}{l}\text { 2. Agelena labyrinthica } \\
\text { (CLERCK) }\end{array}$ & & $\begin{array}{c}0,003 \\
1,3\end{array}$ & $\begin{array}{c}0,004 \\
0,7\end{array}$ & $\begin{array}{c}0,001 \\
0,2\end{array}$ & $\begin{array}{c}0,003 \\
1,2\end{array}$ & & & $\begin{array}{c}0,003 \\
0,7\end{array}$ & & $\begin{array}{c}0,001 \\
0,7\end{array}$ & \\
\hline $\begin{array}{l}\text { 3. Agroeca brunnea } \\
\text { (BLACKW ALL) }\end{array}$ & $\begin{array}{c}0,008 \\
3,1\end{array}$ & $\begin{array}{c}0,007 \\
2,7\end{array}$ & & $\begin{array}{c}0,001 \\
0,2\end{array}$ & $\begin{array}{c}0,008 \\
3,0\end{array}$ & $\begin{array}{c}0,021 \\
6,6\end{array}$ & $\begin{array}{c}0,008 \\
1,3\end{array}$ & $\begin{array}{c}0,016 \\
3,2\end{array}$ & $\begin{array}{c}0,022 \\
8,8\end{array}$ & $\begin{array}{c}0,007 \\
3,9\end{array}$ & $\begin{array}{c}0,016 \\
7,0\end{array}$ \\
\hline $\begin{array}{l}\text { 4. Agroeca cuprea } \\
\text { MENGE }\end{array}$ & & & & & $\begin{array}{c}0,001 \\
0,6 \\
\end{array}$ & & & & & & \\
\hline \begin{tabular}{|l|} 
5. Agroeca proxima \\
(O. P. - CAMBRIDGE) \\
\end{tabular} & $\begin{array}{c}0,002 \\
1,0 \\
\end{array}$ & & & $\begin{array}{c}0,002 \\
0,5\end{array}$ & & & & $\begin{array}{c}0,001 \\
0,3 \\
\end{array}$ & $\begin{array}{c}0,002 \\
1,1 \\
\end{array}$ & $\begin{array}{c}0,014 \\
7,8 \\
\end{array}$ & $\begin{array}{c}0,005 \\
2,3 \\
\end{array}$ \\
\hline $\begin{array}{l}\text { 6. Alopecosa cuneata } \\
\text { (CLERCK) }\end{array}$ & & & & $\begin{array}{c}0,001 \\
0,2 \\
\end{array}$ & $\begin{array}{c}0,001 \\
0,6 \\
\end{array}$ & $\ldots$ & & & & & \\
\hline $\begin{array}{l}\text { 7. Alopecosa pulverentula } \\
\text { (CLERCK) }\end{array}$ & & $\begin{array}{c}0,001 \\
0,6\end{array}$ & $\begin{array}{c}0,008 \\
1,5\end{array}$ & $\begin{array}{c}0,011 \\
2,2\end{array}$ & $\begin{array}{c}0,012 \\
4,3\end{array}$ & $\begin{array}{c}0,001 \\
0,4\end{array}$ & $\begin{array}{c}0,013 \\
2,2\end{array}$ & & & $\begin{array}{c}0,001 \\
0,7\end{array}$ & \\
\hline $\begin{array}{l}\text { 8. Araeoncus crassiceps } \\
\text { (W ESTRING) }\end{array}$ & & & & & & & & & $\begin{array}{c}0,001 \\
0,8\end{array}$ & & \\
\hline $\begin{array}{l}\text { 9. Araeoncus humilis } \\
\text { (BLACKW ALL) }\end{array}$ & & $\begin{array}{c}0,001 \\
0,6\end{array}$ & & & & & & & & & \\
\hline $\begin{array}{l}\text { 10. Bathyphanthes gracilis } \\
\text { (BLACKW ALL) }\end{array}$ & & & & & & & $\begin{array}{c}0,001 \\
0,2\end{array}$ & & & & \\
\hline
\end{tabular}




\begin{tabular}{|c|c|c|c|c|c|c|c|c|c|c|c|}
\hline Fläche & 0 & 0 & 1 & 1 & 1 & 2 & 2 & 2 & 3 & 3 & 3 \\
\hline \begin{tabular}{|l} 
Jahr \\
\end{tabular} & 1998 & 2000 & 1996 & 1998 & 2000 & 1996 & 1998 & 2000 & 1996 & 1998 & 2000 \\
\hline $\begin{array}{l}\text { 11. Centromerus incilium } \\
\text { (L. } \mathrm{KOCH})\end{array}$ & $\begin{array}{c}0,008 \\
3,1\end{array}$ & $\begin{array}{c}0,007 \\
2,7\end{array}$ & $\begin{array}{l}0,027 \\
5,2\end{array}$ & $\begin{array}{c}0,019 \\
3,8\end{array}$ & $\begin{array}{c}0,001 \\
0,6\end{array}$ & & $\begin{array}{c}0,001 \\
0,2\end{array}$ & & & & \\
\hline $\begin{array}{l}\text { 12. Centromerus pabulator } \\
\text { (O. P. - CAMBRIDGE) }\end{array}$ & $\begin{array}{c}0,001 \\
0,5 \\
\end{array}$ & & & & & $\begin{array}{c}0,002 \\
0,8\end{array}$ & $\begin{array}{c}0,001 \\
0,2 \\
\end{array}$ & $\begin{array}{c}0,005 \\
1,0 \\
\end{array}$ & & & $\begin{array}{c}0,007 \\
3,1\end{array}$ \\
\hline $\begin{array}{l}\text { 13. Centromerus sylvaticus } \\
\text { (BLACKWALL) }\end{array}$ & $\begin{array}{c}0,005 \\
2,0\end{array}$ & & & & & $\begin{array}{c}0,002 \\
0,8\end{array}$ & $\begin{array}{c}0,016 \\
2,6\end{array}$ & $\begin{array}{c}0,003 \\
0,7\end{array}$ & $\begin{array}{c}0,002 \\
1,1\end{array}$ & $\begin{array}{c}0,001 \\
0,7\end{array}$ & $\begin{array}{c}0,012 \\
5,5\end{array}$ \\
\hline $\begin{array}{l}\text { 14. Ceratinella brevis } \\
\text { (WIDER) }\end{array}$ & $\begin{array}{c}0,005 \\
2,0 \\
\end{array}$ & $\begin{array}{c}0,007 \\
2,7 \\
\end{array}$ & & & & $\begin{array}{c}0,009 \\
3,0\end{array}$ & $\begin{array}{c}0,001 \\
0,2\end{array}$ & & & & \\
\hline $\begin{array}{l}\text { 15. Cercidia prominens } \\
\text { (WESTRING) }\end{array}$ & $\begin{array}{c}0,002 \\
1,0 \\
\end{array}$ & $\begin{array}{c}0,001 \\
0,6 \\
\end{array}$ & $\begin{array}{l}0,002 \\
0,4\end{array}$ & $\begin{array}{c}0,009 \\
1,9 \\
\end{array}$ & $\begin{array}{c}0,001 \\
0,6 \\
\end{array}$ & & & & & & \\
\hline $\begin{array}{l}\text { 16. Cicurina cicur } \\
\text { (FABR.) }\end{array}$ & & & $\begin{array}{l}0,001 \\
0,2\end{array}$ & $\begin{array}{c}0,001 \\
0,2\end{array}$ & & & $\begin{array}{c}0,002 \\
0,4\end{array}$ & & & & \\
\hline $\begin{array}{l}\text { 17. Clubiona comta } \\
\text { C. L. KOCH }\end{array}$ & & & & & & & & & $\begin{array}{c}0,001 \\
0,5\end{array}$ & & $\begin{array}{c}0,001 \\
0,7\end{array}$ \\
\hline $\begin{array}{l}\text { 18. Clubiona genevensis } \\
\text { L. KOCH }\end{array}$ & & & & & & $\begin{array}{c}0,001 \\
0,4\end{array}$ & & & & & \\
\hline $\begin{array}{l}\text { 19. Clubiona lutescens } \\
\text { WESTRING }\end{array}$ & & & & & & & & & $\begin{array}{c}0,001 \\
0,5\end{array}$ & & \\
\hline $\begin{array}{l}\text { 20. Clubiona terrestris } \\
\text { WESTRING }\end{array}$ & & & & & & $\begin{array}{c}0,001 \\
0,4\end{array}$ & $\begin{array}{c}0,002 \\
0,4\end{array}$ & $\begin{array}{c}0,003 \\
0,7\end{array}$ & & $\begin{array}{c}0,004 \\
2,3\end{array}$ & $\begin{array}{c}0,001 \\
0,7\end{array}$ \\
\hline $\begin{array}{l}\text { 21.Coriarachne depressa } \\
\text { (C. L. } \mathrm{KOCH} \text { ) }\end{array}$ & & & & & & & $\begin{array}{c}0,001 \\
0,1\end{array}$ & $\begin{array}{c}0,001 \\
0,3\end{array}$ & & & \\
\hline $\begin{array}{l}\text { 22. Crustulina guttata } \\
\text { (WIDER) }\end{array}$ & & & $\begin{array}{l}0,001 \\
0,2\end{array}$ & $\begin{array}{c}0,001 \\
0,2\end{array}$ & $\begin{array}{c}0,001 \\
0,6\end{array}$ & & & & $\begin{array}{c}0,001 \\
0,5\end{array}$ & & \\
\hline $\begin{array}{l}\text { 23. Diaea dorsata } \\
\text { (FABR.) }\end{array}$ & & & & & & & & $\begin{array}{c}0,001 \\
0,3 \\
\end{array}$ & & & \\
\hline
\end{tabular}


Tab. 1 Forts./Tab. 1 cont.

\begin{tabular}{|c|c|c|c|c|c|c|c|c|c|c|c|}
\hline Fläche & 0 & 0 & 1 & 1 & 1 & 2 & 2 & 2 & 3 & 3 & 3 \\
\hline Jahr & 1998 & 2000 & 1996 & 1998 & 2000 & 1996 & 1998 & 2000 & 1996 & 1998 & 2000 \\
\hline $\begin{array}{l}\text { 24. Dictyna pusilla } \\
\text { THORELL }\end{array}$ & & & & $\begin{array}{c}0,001 \\
0,2\end{array}$ & & & & & & & \\
\hline $\begin{array}{l}\text { 25. Dicymbium nigrum } \\
\text { (BLACKWALL) }\end{array}$ & & & $\begin{array}{l}0,002 \\
0,4\end{array}$ & $\begin{array}{c}0,008 \\
1,6\end{array}$ & & & $\begin{array}{c}0,006 \\
1,1\end{array}$ & & & & \\
\hline $\begin{array}{l}\text { 26. Diplostyla concolor } \\
\text { (WIDER) }\end{array}$ & & & $\begin{array}{l}0,002 \\
0,4\end{array}$ & $\begin{array}{c}0,008 \\
1,6\end{array}$ & & & $\begin{array}{c}0,009 \\
1,5\end{array}$ & & & & \\
\hline $\begin{array}{l}\text { 27. Drapetisca socialis } \\
\text { (SUNDEVALL) }\end{array}$ & & & & & & $\begin{array}{c}0,001 \\
0,4\end{array}$ & & & & & \\
\hline $\begin{array}{l}\text { 28. Drassyllus praeficus } \\
\text { (L. KOCH) }\end{array}$ & & & & $\begin{array}{c}0,009 \\
1,9\end{array}$ & & & $\begin{array}{c}0,001 \\
0,2 \\
\end{array}$ & & & & \\
\hline $\begin{array}{l}\text { 29. Drassyllus pusillus } \\
\text { (C. L. KOCH) }\end{array}$ & & & & $\begin{array}{c}0,001 \\
0,2\end{array}$ & & & $\begin{array}{c}0,002 \\
0,4\end{array}$ & & & & \\
\hline $\begin{array}{l}\text { 30. Enoplognatha thoracica } \\
\text { (HAHN) }\end{array}$ & & & & & $\begin{array}{c}0,005 \\
1,8\end{array}$ & & & $\begin{array}{c}0,001 \\
0,3\end{array}$ & & & \\
\hline $\begin{array}{l}\text { 31. Erigone atra } \\
\text { BLACKWALL }\end{array}$ & & & & & & & $\begin{array}{c}0,001 \\
0,2\end{array}$ & $\begin{array}{c}0,001 \\
0,3\end{array}$ & & & \\
\hline $\begin{array}{l}\text { 32. Ero furcata } \\
\text { (VILLERS) }\end{array}$ & $\begin{array}{c}0,002 \\
1,0\end{array}$ & $\begin{array}{c}0,003 \\
1,3\end{array}$ & & & & & $\begin{array}{c}0,001 \\
0,2\end{array}$ & & & & \\
\hline $\begin{array}{l}\text { 33. Euophrys erratica } \\
\text { (WALCKENAER) }\end{array}$ & & & & & & & & & & $\begin{array}{c}0,001 \\
0,7\end{array}$ & \\
\hline $\begin{array}{l}\text { 34. Euophrys frontalis } \\
\text { (WALCKENAER) }\end{array}$ & $\begin{array}{c}0,001 \\
0,5\end{array}$ & $\begin{array}{c}0,005 \\
2,0\end{array}$ & $\begin{array}{l}0,004 \\
0,7\end{array}$ & $\begin{array}{c}0,016 \\
3,3\end{array}$ & $\begin{array}{c}0,008 \\
3,0\end{array}$ & $\begin{array}{c}0,001 \\
0,4\end{array}$ & $\begin{array}{c}0,002 \\
0,4 \\
\end{array}$ & $\begin{array}{c}0,007 \\
1,4 \\
\end{array}$ & $\begin{array}{c}0,001 \\
0,5\end{array}$ & & $\begin{array}{c}0,001 \\
0,7 \\
\end{array}$ \\
\hline $\begin{array}{l}\text { 35. Euryopis flavomaculata } \\
\text { (C. L. KOCH) }\end{array}$ & $\begin{array}{c}0,017 \\
6,3\end{array}$ & $\begin{array}{c}0,050 \\
18,9\end{array}$ & $\begin{array}{l}0,029 \\
5,5\end{array}$ & $\begin{array}{c}0,058 \\
11,5\end{array}$ & $\begin{array}{c}0,062 \\
21,3\end{array}$ & $\begin{array}{c}0,014 \\
4,4\end{array}$ & $\begin{array}{c}0,088 \\
14,4\end{array}$ & $\begin{array}{c}0,069 \\
14,0\end{array}$ & $\begin{array}{c}0,011 \\
4,4\end{array}$ & $\begin{array}{c}0,002 \\
1,5\end{array}$ & $\begin{array}{c}0,003 \\
1,6\end{array}$ \\
\hline
\end{tabular}




\begin{tabular}{|c|c|c|c|c|c|c|c|c|c|c|c|}
\hline Fläche & 0 & 0 & 1 & 1 & 1 & 2 & 2 & 2 & 3 & 3 & 3 \\
\hline Jahr & 1998 & 2000 & 1996 & 1998 & 2000 & 1996 & 1998 & 2000 & 1996 & 1998 & 2000 \\
\hline $\begin{array}{l}\text { 36. Evarcha falcata } \\
\text { (CLERCK) }\end{array}$ & & $\begin{array}{c}0,001 \\
0,6 \\
\end{array}$ & $\begin{array}{l}0,002 \\
0,4 \\
\end{array}$ & $\begin{array}{c}0,004 \\
0,8\end{array}$ & & & $\begin{array}{c}0,002 \\
0,4 \\
\end{array}$ & & & & \\
\hline $\begin{array}{l}\text { 37. Gongylidiellum latebricola } \\
\text { (O. P. - CAMBRIDGE) }\end{array}$ & & $\begin{array}{c}0,003 \\
1,3 \\
\end{array}$ & & $\begin{array}{c}0,001 \\
0,2 \\
\end{array}$ & & & & & & & \\
\hline $\begin{array}{l}\text { 38. Gongylidiellum murcidum } \\
\text { SIMON }\end{array}$ & & & & & & $\begin{array}{c}0,001 \\
0,4\end{array}$ & & & & & \\
\hline $\begin{array}{l}\text { 39. Haplodrassus signifer } \\
\text { (C. L. KOCH) }\end{array}$ & $\begin{array}{c}0,001 \\
0,5\end{array}$ & $\begin{array}{c}0,003 \\
1,3\end{array}$ & $\begin{array}{l}0,008 \\
1,5\end{array}$ & $\begin{array}{c}0,009 \\
1,9\end{array}$ & $\begin{array}{c}0,003 \\
1,2\end{array}$ & & $\begin{array}{c}0,001 \\
0,2\end{array}$ & $\begin{array}{c}0,001 \\
0,3\end{array}$ & & & \\
\hline $\begin{array}{l}\text { 40. Haplodrassus silvestris } \\
\text { (BLACKWALL) }\end{array}$ & & & & $\begin{array}{c}0,001 \\
0,2\end{array}$ & & $\begin{array}{c}0,002 \\
0,8 \\
\end{array}$ & $\begin{array}{c}0,006 \\
1,1 \\
\end{array}$ & $\begin{array}{c}0,008 \\
1,8\end{array}$ & & $\begin{array}{c}0,001 \\
0,7 \\
\end{array}$ & \\
\hline $\begin{array}{l}\text { 41. Haplodrassus soerenseni } \\
\text { (STRAND) }\end{array}$ & $\begin{array}{c}0,008 \\
3,1 \\
\end{array}$ & & & $\begin{array}{c}0,002 \\
0,5\end{array}$ & $\begin{array}{c}0,001 \\
0,6 \\
\end{array}$ & $\begin{array}{c}0,001 \\
0,4 \\
\end{array}$ & $\begin{array}{c}0,012 \\
1,9 \\
\end{array}$ & $\begin{array}{c}0,007 \\
1,4\end{array}$ & & $\begin{array}{r}0,007 \\
3,9 \\
\end{array}$ & $\begin{array}{c}0,010 \\
4,7 \\
\end{array}$ \\
\hline $\begin{array}{l}\text { 42. Haplodrassus umbratilis } \\
\text { (L. KOCH) }\end{array}$ & & & $\begin{array}{l}0,002 \\
0,2 \\
\end{array}$ & $\begin{array}{c}0,008 \\
1,6 \\
\end{array}$ & $\begin{array}{c}0,005 \\
1,8 \\
\end{array}$ & & & $\begin{array}{c}0,007 \\
1,4 \\
\end{array}$ & & & $\begin{array}{c}0,001 \\
0,7 \\
\end{array}$ \\
\hline $\begin{array}{l}\text { 43. Harpactea rubicunda } \\
\text { (C. L. KOCH) }\end{array}$ & & & & $\begin{array}{c}0,002 \\
0,5 \\
\end{array}$ & $\begin{array}{c}0,001 \\
0,6 \\
\end{array}$ & & $\begin{array}{c}0,001 \\
0,2 \\
\end{array}$ & & & & \\
\hline $\begin{array}{l}\text { 44. Lepthyphantes angulipalpis } \\
\text { (WESTRING) }\end{array}$ & & & & & & $\begin{array}{c}0,002 \\
0,8 \\
\end{array}$ & & & $\begin{array}{c}0,002 \\
1,1 \\
\end{array}$ & $\begin{array}{c}0,001 \\
0,7 \\
\end{array}$ & $\begin{array}{c}0,001 \\
0,7 \\
\end{array}$ \\
\hline $\begin{array}{l}\text { 45. Lepthyphantes flavipes } \\
\text { (BLACKWALL) }\end{array}$ & $\begin{array}{c}0,030 \\
10,9\end{array}$ & $\begin{array}{c}0,008 \\
3,4\end{array}$ & $\begin{array}{l}0,004 \\
0,7\end{array}$ & $\begin{array}{c}0,001 \\
0,2\end{array}$ & $\begin{array}{c}0,001 \\
0,6\end{array}$ & $\begin{array}{c}0,052 \\
16,2 \\
\end{array}$ & $\begin{array}{c}0,068 \\
11,0\end{array}$ & $\begin{array}{c}0,043 \\
8,6\end{array}$ & $\begin{array}{c}0,044 \\
17,6 \\
\end{array}$ & $\begin{array}{c}0,055 \\
29,7 \\
\end{array}$ & $\begin{array}{r}0,073 \\
32,3 \\
\end{array}$ \\
\hline $\begin{array}{l}\text { 46. Lepthyphantes mansuetus } \\
\text { (THORELL) }\end{array}$ & & $\begin{array}{c}0,001 \\
0,6 \\
\end{array}$ & $\begin{array}{l}0,005 \\
1,0 \\
\end{array}$ & $\begin{array}{c}0,001 \\
0,2 \\
\end{array}$ & & $\begin{array}{c}0,002 \\
0,8 \\
\end{array}$ & $\begin{array}{c}0,002 \\
0,4 \\
\end{array}$ & & $\begin{array}{c}0,002 \\
1,1\end{array}$ & & \\
\hline $\begin{array}{l}\text { 47. Lepthyphantes mengei } \\
\text { KULCZYNSKI }\end{array}$ & $\begin{array}{c}0,001 \\
0,5 \\
\end{array}$ & & & & & & & & & & \\
\hline $\begin{array}{l}\text { 48. Lepthyphantes pallidus } \\
\text { (O. P. -CAMBRIDGE) }\end{array}$ & $\begin{array}{c}0,001 \\
0,5 \\
\end{array}$ & & & & & $\begin{array}{c}0,001 \\
0,4 \\
\end{array}$ & $\begin{array}{c}0,001 \\
0,2 \\
\end{array}$ & & & & $\begin{array}{c}0,001 \\
0,7 \\
\end{array}$ \\
\hline
\end{tabular}


Tab. 1 Forts./Tab. 1 cont.

\begin{tabular}{|c|c|c|c|c|c|c|c|c|c|c|c|}
\hline Fläche & 0 & 0 & 1 & 1 & 1 & 2 & 2 & 2 & 3 & 3 & 3 \\
\hline Jahr & 1998 & 2000 & 1996 & 1998 & 2000 & 1996 & 1998 & 2000 & 1996 & 1998 & 2000 \\
\hline $\begin{array}{l}\text { 49. Lepthyphantes tenuis } \\
\text { (BLACKWALL) }\end{array}$ & & $\begin{array}{c}0,001 \\
0,6\end{array}$ & $\begin{array}{l}0,002 \\
0,2 \\
\end{array}$ & & & & & & & & \\
\hline $\begin{array}{l}\text { 50. Linyphia hortensis } \\
\text { SUNDEVALL }\end{array}$ & & & & & & & $\begin{array}{c}0,001 \\
0,2 \\
\end{array}$ & & & 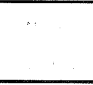 & \\
\hline $\begin{array}{l}\text { 51. Linyphia triangularis } \\
\text { (CLERCK) }\end{array}$ & & & $\begin{array}{l}0,002 \\
0,2 \\
\end{array}$ & & & & $\begin{array}{c}0,001 \\
0,2 \\
\end{array}$ & & & & \\
\hline $\begin{array}{l}\text { 52. Macrargus carpenteri } \\
\text { (O. P. -CAMBRIDGE) }\end{array}$ & $\begin{array}{c}0,005 \\
0,5\end{array}$ & & & $\begin{array}{c}0,002 \\
0,2\end{array}$ & & & & & & & \\
\hline $\begin{array}{l}\text { 53. Macrargus rufus } \\
\text { (WIDER) }\end{array}$ & & $\begin{array}{c}0,003 \\
1,3 \\
\end{array}$ & & & $\begin{array}{c}0,003 \\
1,2 \\
\end{array}$ & $\begin{array}{c}0,002 \\
0,8\end{array}$ & & $\begin{array}{c}0,001 \\
0,3 \\
\end{array}$ & $\begin{array}{c}0,014 \\
5,5\end{array}$ & & $\begin{array}{c}0,005 \\
2,3 \\
\end{array}$ \\
\hline $\begin{array}{l}\text { 54. Meioneta rurestris } \\
\text { (C. L. KOCH) }\end{array}$ & & & $\begin{array}{ll}0,002 \\
0,2 \\
\end{array}$ & & & & & & & & \\
\hline $\begin{array}{l}\text { 55. Micaria fulgens } \\
\text { (WALCKENAER) }\end{array}$ & & & & & & & $\begin{array}{c}0,001 \\
0,2 \\
\end{array}$ & & & & \\
\hline $\begin{array}{l}\text { 56. Micaria pulicaria } \\
\text { (SUNDEVALL) }\end{array}$ & & & & & & & $\begin{array}{c}0,001 \\
0,2\end{array}$ & & & & \\
\hline $\begin{array}{l}\text { 57. Micrargus herbigradus } \\
\text { (BLACKWALL) }\end{array}$ & & $\begin{array}{c}0,001 \\
0,6\end{array}$ & & $\begin{array}{c}0,004 \\
0,8\end{array}$ & $\begin{array}{c}0,008 \\
3,0\end{array}$ & $\begin{array}{c}0,001 \\
0,4\end{array}$ & & & & & \\
\hline $\begin{array}{l}\text { 58. Microneta viaria } \\
\text { (BLACKWALL) }\end{array}$ & & & & & & & $\begin{array}{c}0,002 \\
0,4 \\
\end{array}$ & $\begin{array}{c}0,001 \\
0,3 \\
\end{array}$ & $\begin{array}{c}0,005 \\
2,2 \\
\end{array}$ & & $\begin{array}{c}0,001 \\
0,7 \\
\end{array}$ \\
\hline $\begin{array}{l}\text { 59. Neon reticulatus } \\
\text { (BLACKWALL) }\end{array}$ & $\begin{array}{c}0,001 \\
0,5\end{array}$ & & $\begin{array}{l}0,004 \\
0,4 \\
\end{array}$ & & & & & & $\begin{array}{c}0,001 \\
0,5\end{array}$ & $\begin{array}{c}0,004 \\
2,2\end{array}$ & \\
\hline $\begin{array}{l}\text { 60. Neottiura bimaculata } \\
\text { (L.) }\end{array}$ & $\begin{array}{c}0,001 \\
0,5 \\
\end{array}$ & & & $\begin{array}{c}0,001 \\
0,2\end{array}$ & & & $\begin{array}{c}0,002 \\
0,4 \\
\end{array}$ & $\begin{array}{c}0,001 \\
0,3 \\
\end{array}$ & & & \\
\hline
\end{tabular}




\begin{tabular}{|c|c|c|c|c|c|c|c|c|c|c|c|}
\hline Fläche & 0 & 0 & 1 & 1 & 1 & 2 & 2 & 2 & 3 & 3 & 3 \\
\hline Jahr & 1998 & 2000 & 1996 & 1998 & 2000 & 1996 & 1998 & 2000 & 1996 & 1998 & 2000 \\
\hline 61. Neriene clathrata & 0,002 & 0,001 & 0,002 & 0,001 & & 0,004 & 0,004 & & 0,001 & & \\
\hline$\left(\frac{\text { SUNDEVALL })}{62 \text { Oedothorax retusus }}\right.$ & & & $\frac{0,2}{0,002}$ & $\frac{0,2}{0,001}$ & & & 0,6 & & & & \\
\hline (WESTRING) & & & 0,2 & $\begin{array}{c}, 001 \\
0,2\end{array}$ & & & & & & & \\
\hline $\begin{array}{l}\text { 63. Ozyptila praticola } \\
\text { (C. L. KOCH) }\end{array}$ & & & & & & $\begin{array}{c}0,002 \\
0,8\end{array}$ & & $\begin{array}{c}0,001 \\
0,3\end{array}$ & & & $\begin{array}{c}0,003 \\
1,6\end{array}$ \\
\hline $\begin{array}{l}\text { 64. Pachygnatha clercki } \\
\text { SUNDEVALL }\end{array}$ & $\begin{array}{c}0,002 \\
1,0\end{array}$ & & $\begin{array}{l}0,004 \\
0,4\end{array}$ & $\begin{array}{c}0,001 \\
0,2\end{array}$ & & $\begin{array}{c}0,001 \\
0,4\end{array}$ & & & & & \\
\hline $\begin{array}{l}\text { 65. Pachygnatha degeeri } \\
\text { SUNDEVALL }\end{array}$ & & & $\begin{array}{l}0,004 \\
0,4\end{array}$ & & & $\begin{array}{c}0,004 \\
1,3\end{array}$ & & & & & \\
\hline $\begin{array}{l}\text { 66. Pardosa amentata } \\
\text { (CLERCK) }\end{array}$ & & & & & & $\begin{array}{c}0,002 \\
0,8\end{array}$ & & & & & \\
\hline $\begin{array}{l}\text { 67. Pardosa lugubris } \\
\text { (WALCKENAER) }\end{array}$ & $\begin{array}{c}0,001 \\
0,5\end{array}$ & & & $\begin{array}{c}0,001 \\
0,2\end{array}$ & $\begin{array}{c}0,001 \\
0,6\end{array}$ & & & & $\begin{array}{c}0,001 \\
0,5 \\
\end{array}$ & & \\
\hline $\begin{array}{l}\text { 68. Pardosa prativaga } \\
\text { (L. KOCH) }\end{array}$ & & $\begin{array}{c}0,001 \\
0,6\end{array}$ & & $\begin{array}{c}0,004 \\
0,8\end{array}$ & $\begin{array}{c}0,001 \\
0,6\end{array}$ & & $\begin{array}{c}0,001 \\
0,2\end{array}$ & $\begin{array}{c}0,001 \\
0,3\end{array}$ & & & $\begin{array}{c}0,003 \\
1,6\end{array}$ \\
\hline $\begin{array}{l}\text { 69. Pardosa saltans } \\
\text { TÖPFER-HOFMANN }\end{array}$ & & & & $\begin{array}{c}0,001 \\
0,2\end{array}$ & & & $\begin{array}{c}0,001 \\
0,2\end{array}$ & $\begin{array}{c}0,007 \\
1,4\end{array}$ & & & $\begin{array}{c}0,008 \\
3,9\end{array}$ \\
\hline $\begin{array}{l}\text { 70. Pelecopsis radicicola } \\
\text { (L. KOCH) }\end{array}$ & $\begin{array}{c}0,001 \\
0,5\end{array}$ & $\begin{array}{c}0,012 \\
4,7\end{array}$ & $\begin{array}{l}0,007 \\
1,3\end{array}$ & $\begin{array}{c}0,008 \\
1,6\end{array}$ & $\begin{array}{c}0,001 \\
0,6\end{array}$ & $\begin{array}{c}0,027 \\
8,3\end{array}$ & $\begin{array}{c}0,027 \\
4,5\end{array}$ & $\begin{array}{c}0,121 \\
24,5\end{array}$ & & & $\begin{array}{c}0,003 \\
1,6\end{array}$ \\
\hline $\begin{array}{l}\text { 71. Philodromus collinus } \\
\text { C. L. KOCH }\end{array}$ & & & $\begin{array}{l}0,004 \\
0,4\end{array}$ & & & $\begin{array}{c}0,002 \\
0,8\end{array}$ & $\begin{array}{c}0,001 \\
0,2\end{array}$ & & $\begin{array}{c}0,001 \\
0,5\end{array}$ & & \\
\hline $\begin{array}{l}\text { 72. Philodromus fuscomarginatus } \\
\text { (DEGEER) }\end{array}$ & & & $\begin{array}{l}0,002 \\
0,2\end{array}$ & & & & & & & $\begin{array}{c}0,001 \\
0,7 \\
\end{array}$ & \\
\hline $\begin{array}{l}\text { 73. Philodromus margaritatus } \\
\text { (CLERCK) }\end{array}$ & & $\begin{array}{c}0,001 \\
0,6\end{array}$ & & & & & & & & & \\
\hline
\end{tabular}


Tab. 1 Forts./Tab. 1 cont.

\begin{tabular}{|c|c|c|c|c|c|c|c|c|c|c|c|}
\hline Fläche & 0 & 0 & 1 & 1 & 1 & 2 & 2 & 2 & 3 & 3 & 3 \\
\hline Jahr & 1998 & 2000 & 1996 & 1998 & 2000 & 1996 & 1998 & 2000 & 1996 & 1998 & 2000 \\
\hline $\begin{array}{l}\text { 74. Pholcomma gibbum } \\
\text { (WESTRING) }\end{array}$ & & $\begin{array}{c}0,001 \\
0,6\end{array}$ & & & & & & & & & \\
\hline $\begin{array}{l}\text { 75. Phrurolithus festivus } \\
\text { (C. L. KOCH) }\end{array}$ & & & $\begin{array}{l}0,036 \\
6,8\end{array}$ & $\begin{array}{c}0,008 \\
1,6\end{array}$ & $\begin{array}{c}0,001 \\
0,6\end{array}$ & $\begin{array}{c}0,001 \\
0,4\end{array}$ & $\begin{array}{c}0,014 \\
2,2\end{array}$ & $\begin{array}{c}0,007 \\
1,4\end{array}$ & & & \\
\hline $\begin{array}{l}\text { 76. Pirata hygrophilus } \\
\text { THORELL }\end{array}$ & $\begin{array}{c}0,001 \\
0,5\end{array}$ & & & & & & & & & & \\
\hline $\begin{array}{l}\text { 77. Pisaura mirabilis } \\
\text { (CLERCK) }\end{array}$ & & & & $\begin{array}{c}0,001 \\
0,2\end{array}$ & & & $\begin{array}{c}0,001 \\
0,2\end{array}$ & & & & \\
\hline $\begin{array}{l}\text { 78. Pocadicnemis pumila } \\
\text { (BLACKWALL) }\end{array}$ & $\begin{array}{c}0,012 \\
4,7\end{array}$ & $\begin{array}{c}0,005 \\
2,0\end{array}$ & \begin{tabular}{l|}
0,009 \\
1,8 \\
\end{tabular} & $\begin{array}{c}0,026 \\
5,2\end{array}$ & $\begin{array}{c}0,001 \\
0,6\end{array}$ & $\begin{array}{c}0,011 \\
3,5\end{array}$ & $\begin{array}{c}0,006 \\
1,1\end{array}$ & & & & \\
\hline $\begin{array}{l}\text { 79. Robertus lividus } \\
\text { (BLACKWALL) }\end{array}$ & $\begin{array}{c}0,014 \\
5,2\end{array}$ & & $\begin{array}{l}0,004 \\
0,7 \\
\end{array}$ & $\begin{array}{c}0,005 \\
1,0\end{array}$ & & $\begin{array}{c}0,007 \\
2,2\end{array}$ & & $\begin{array}{c}0,001 \\
0,3\end{array}$ & $\begin{array}{c}0,004 \\
1,6\end{array}$ & $\begin{array}{c}0,002 \\
1,5\end{array}$ & \\
\hline $\begin{array}{l}\text { 80. Saaristoa abnormis } \\
\text { (BLACKWALL) }\end{array}$ & $\begin{array}{c}0,007 \\
2,6 \\
\end{array}$ & $\begin{array}{c}0,001 \\
0,6\end{array}$ & & & & $\begin{array}{c}0,004 \\
1,3 \\
\end{array}$ & & & & & \\
\hline $\begin{array}{l}\text { 81. Salticus zebraneus } \\
\text { (C. L. KOCH) }\end{array}$ & & & & & & & & & & $\begin{array}{c}0,001 \\
0,7\end{array}$ & $\begin{array}{c}0,001 \\
0,7 \\
\end{array}$ \\
\hline $\begin{array}{l}\text { 82. Scotina palliardi } \\
\text { (L. KOCH) }\end{array}$ & & & \begin{tabular}{|l|}
0,002 \\
0,2 \\
\end{tabular} & & & & & & & & \\
\hline $\begin{array}{l}\text { 83. Segestria senoculata } \\
\text { (L.) }\end{array}$ & $\begin{array}{c}0,004 \\
1,6 \\
\end{array}$ & $\begin{array}{c}0,001 \\
0,6\end{array}$ & \begin{tabular}{|l|}
0,002 \\
0,2 \\
\end{tabular} & & & & & $\begin{array}{c}0,007 \\
1,4 \\
\end{array}$ & & & $\begin{array}{c}0,003 \\
1,6 \\
\end{array}$ \\
\hline $\begin{array}{l}\text { 84. Steatodea phalerata } \\
\text { (PANZER) }\end{array}$ & & $\begin{array}{c}0,003 \\
1,3 \\
\end{array}$ & \begin{tabular}{|l|}
0,004 \\
0,4 \\
\end{tabular} & $\begin{array}{c}0,002 \\
0,5\end{array}$ & $\begin{array}{c}0,010 \\
3,6\end{array}$ & & & & & & \\
\hline $\begin{array}{l}\text { 85. Stemonyphanthes lineatus } \\
\text { (L.) }\end{array}$ & & $\begin{array}{c}0,001 \\
0,6\end{array}$ & & & & & & & & & \\
\hline
\end{tabular}




\begin{tabular}{|c|c|c|c|c|c|c|c|c|c|c|c|}
\hline Fläche & 0 & 0 & 1 & 1 & 1 & 2 & 2 & 2 & 3 & 3 & 3 \\
\hline \begin{tabular}{|l|} 
Jahr \\
\end{tabular} & 1998 & 2000 & 1996 & 1998 & 2000 & 1996 & 1998 & 2000 & 1996 & 1998 & 2000 \\
\hline $\begin{array}{l}\text { 86. Tapinocyba praecox } \\
\text { (O. P. -CAMBRIDGE) }\end{array}$ & & & $\begin{array}{l}0,026 \\
4,9\end{array}$ & & $\begin{array}{c}0,003 \\
1,2\end{array}$ & $\begin{array}{c}0,002 \\
0,8\end{array}$ & & & $\begin{array}{c}0,006 \\
2,7\end{array}$ & $\begin{array}{c}0,001 \\
0,7\end{array}$ & \\
\hline \begin{tabular}{|l} 
87. Tapinopa longidens \\
(WIDER)
\end{tabular} & & & & & & $\begin{array}{c}0,001 \\
0,4\end{array}$ & & & & & \\
\hline \begin{tabular}{|l} 
88. Tetragnatha pinicola \\
L. KOCH \\
\end{tabular} & & & & & $\begin{array}{c}0,001 \\
0,6 \\
\end{array}$ & & & & & & \\
\hline $\begin{array}{l}\text { 89. Trochosa terricola } \\
\text { THORELL } \\
\end{array}$ & $\begin{array}{c}0,017 \\
6,3 \\
\end{array}$ & $\begin{array}{c}0,008 \\
3,4 \\
\end{array}$ & $\begin{array}{l}0,069 \\
13,0 \\
\end{array}$ & $\begin{array}{c}0,023 \\
4,6 \\
\end{array}$ & $\begin{array}{c}0,032 \\
10,9 \\
\end{array}$ & $\begin{array}{c}0,008 \\
2,6 \\
\end{array}$ & $\begin{array}{c}0,088 \\
14,4 \\
\end{array}$ & $\begin{array}{c}0,025 \\
5,0 \\
\end{array}$ & $\begin{array}{c}0,006 \\
2,7 \\
\end{array}$ & $\begin{array}{c}0,057 \\
30,4 \\
\end{array}$ & $\begin{array}{c}0,014 \\
6,3 \\
\end{array}$ \\
\hline $\begin{array}{l}\text { 90. Walckenaeria antica } \\
\text { (WIDER) }\end{array}$ & & & $\begin{array}{l}0,002 \\
0,2 \\
\end{array}$ & & $\begin{array}{c}0,001 \\
0,6\end{array}$ & & & & & & \\
\hline $\begin{array}{l}\text { 91. Walckenaeria atrotibialis } \\
\text { (O. P. -CAMBRIDGE) }\end{array}$ & $\begin{array}{c}0,010 \\
3,6\end{array}$ & & $\begin{array}{l}0,059 \\
11,2\end{array}$ & $\begin{array}{c}0,008 \\
1,6\end{array}$ & & $\begin{array}{c}0,039 \\
12,2\end{array}$ & $\begin{array}{c}0,016 \\
2,6\end{array}$ & $\begin{array}{c}0,014 \\
2,8\end{array}$ & $\begin{array}{c}0,027 \\
11,0\end{array}$ & & \\
\hline $\begin{array}{l}\text { 92. Walckenaeria cucullata } \\
\text { (C. L. } \mathrm{KOCH} \text { ) }\end{array}$ & & & $\begin{array}{ll}0,023 \\
4,4\end{array}$ & $\begin{array}{c}0,005 \\
1,0\end{array}$ & $\begin{array}{c}0,005 \\
1,8\end{array}$ & $\begin{array}{c}0,017 \\
5,2\end{array}$ & $\begin{array}{c}0,006 \\
1,1\end{array}$ & $\begin{array}{c}0,005 \\
1,0\end{array}$ & $\begin{array}{c}0,020 \\
8,3\end{array}$ & $\begin{array}{c}0,001 \\
0,7\end{array}$ & \\
\hline $\begin{array}{l}\text { 93. Walckenaeria dysderoides } \\
\text { (WIDER) }\end{array}$ & $\begin{array}{c}0,008 \\
3,1\end{array}$ & $\begin{array}{c}0,007 \\
2,7\end{array}$ & $\begin{array}{l}0,038 \\
7,3\end{array}$ & $\begin{array}{c}0,015 \\
3,0\end{array}$ & $\begin{array}{c}0,001 \\
0,6\end{array}$ & $\begin{array}{c}0,018 \\
5,7\end{array}$ & $\begin{array}{c}0,005 \\
0,8\end{array}$ & $\begin{array}{c}0,005 \\
1,0\end{array}$ & $\begin{array}{c}0,015 \\
6,0 \\
\end{array}$ & $\begin{array}{c}0,002 \\
1,5\end{array}$ & $\begin{array}{c}0,001 \\
0,7\end{array}$ \\
\hline $\begin{array}{l}\text { 94. Walckenaeria furcillata } \\
\text { (MENGE) }\end{array}$ & $\begin{array}{c}0,021 \\
7,8\end{array}$ & $\begin{array}{c}0,030 \\
11.5\end{array}$ & $\begin{array}{l}0,011 \\
2,0\end{array}$ & $\begin{array}{c}0,016 \\
3,3\end{array}$ & $\begin{array}{c}0,001 \\
0,6\end{array}$ & $\begin{array}{c}0,008 \\
2,6\end{array}$ & $\begin{array}{c}0,011 \\
1,7\end{array}$ & $\begin{array}{c}0,026 \\
5,4\end{array}$ & $\begin{array}{c}0,015 \\
6,0\end{array}$ & $\begin{array}{c}0,001 \\
0,7\end{array}$ & $\begin{array}{c}0,010 \\
4,7\end{array}$ \\
\hline $\begin{array}{l}\text { 95. Walckenaeria obtusa } \\
\text { BLACKWALL }\end{array}$ & & & $\begin{array}{l}0,002 \\
0,2 \\
\end{array}$ & $\begin{array}{c}0,001 \\
0,2 \\
\end{array}$ & & $\begin{array}{c}0,004 \\
1,3 \\
\end{array}$ & $\begin{array}{c}0,002 \\
0,4 \\
\end{array}$ & & & & \\
\hline $\begin{array}{l}\text { 96. Xerolycosa nemoralis } \\
\text { (WESTRING) }\end{array}$ & & & & $\begin{array}{c}0,057 \\
11,2 \\
\end{array}$ & & & $\begin{array}{c}0,025 \\
4,0 \\
\end{array}$ & & & & \\
\hline $\begin{array}{l}\text { 97. Xysticus audax } \\
\text { (SCHRANK) }\end{array}$ & & & \begin{tabular}{|l|}
0,004 \\
0,7
\end{tabular} & & & $\begin{array}{c}0,001 \\
0,4 \\
\end{array}$ & & & & & \\
\hline $\begin{array}{l}\text { 98. Xysticus cristatus } \\
\text { (CLERCK) }\end{array}$ & & & & & & & $\begin{array}{c}0,001 \\
0,2 \\
\end{array}$ & & & & \\
\hline
\end{tabular}


Tab. 1 Forts./Tab. 1 cont.

\begin{tabular}{|c|c|c|c|c|c|c|c|c|c|c|c|}
\hline Fläche & 0 & 0 & 1 & 1 & 1 & 2 & 2 & 2 & 3 & 3 & 3 \\
\hline Jahr & 1998 & 2000 & 1996 & 1998 & 2000 & 1996 & 1998 & 2000 & 1996 & 1998 & 2000 \\
\hline $\begin{array}{l}\text { 99. Xysticus kochi } \\
\text { THORELL }\end{array}$ & & & & $\begin{array}{c}0,001 \\
0,2\end{array}$ & $\begin{array}{c}0,005 \\
1,8\end{array}$ & & & & & & \\
\hline $\begin{array}{l}\text { 100. Xysticus luctuosus } \\
\text { (BLA CKW ALL) }\end{array}$ & $\begin{array}{c}0,001 \\
0,5 \\
\end{array}$ & & & & & & & & & & \\
\hline $\begin{array}{l}\text { 101. Zelotes clivicola } \\
\text { (L. } \mathrm{KOCH})\end{array}$ & $\begin{array}{c}0,005 \\
2,0\end{array}$ & $\begin{array}{c}0,032 \\
12,1 \\
\end{array}$ & $\begin{array}{l}0,047 \\
8,8 \\
\end{array}$ & $\begin{array}{l}0,050 \\
9,9\end{array}$ & $\begin{array}{c}0,053 \\
18,3 \\
\end{array}$ & & $\begin{array}{c}0,019 \\
3,1\end{array}$ & $\begin{array}{c}0,003 \\
0,7\end{array}$ & & & $\begin{array}{c}0,001 \\
0,7\end{array}$ \\
\hline $\begin{array}{l}\text { 102. Zelotes longipes } \\
\text { (L. KOCH) }\end{array}$ & & , & & & & & $\begin{array}{c}0,001 \\
0,2\end{array}$ & & & & \\
\hline $\begin{array}{l}\text { 103. Zelotes petrensis } \\
\text { (C. L. KOCH) }\end{array}$ & & & & & & & $\begin{array}{c}0,002 \\
0,4\end{array}$ & & & & \\
\hline $\begin{array}{l}\text { 104. Zelotes subterraneus } \\
\text { (C. L. } \mathrm{KOCH} \text { ) }\end{array}$ & $\begin{array}{c}0,008 \\
3,1\end{array}$ & $\begin{array}{c}0,025 \\
9,4\end{array}$ & $\begin{array}{l}0,041 \\
7,8\end{array}$ & $\begin{array}{c}0,042 \\
8,2\end{array}$ & $\begin{array}{c}0,023 \\
7,9\end{array}$ & $\begin{array}{c}0,001 \\
0,4\end{array}$ & $\begin{array}{c}0,055 \\
8,9\end{array}$ & $\begin{array}{c}0,036 \\
7,2\end{array}$ & $\begin{array}{c}0,009 \\
3,8\end{array}$ & $\begin{array}{c}0,005 \\
3,1\end{array}$ & $\begin{array}{c}0,016 \\
7,0\end{array}$ \\
\hline $\begin{array}{l}\text { 105. Zora spinimana } \\
\text { (SUNDEVALL) }\end{array}$ & $\begin{array}{c}0,040 \\
14,6\end{array}$ & $\begin{array}{c}0,012 \\
4,7\end{array}$ & $\begin{array}{l}0,016 \\
3,1\end{array}$ & $\begin{array}{c}0,021 \\
4,1\end{array}$ & $\begin{array}{c}0,003 \\
1,2\end{array}$ & $\begin{array}{c}0,009 \\
3,0\end{array}$ & $\begin{array}{c}0,037 \\
6,0\end{array}$ & $\begin{array}{c}0,026 \\
5,4\end{array}$ & $\begin{array}{c}0,016 \\
6,6\end{array}$ & & $\begin{array}{c}0,010 \\
4,7\end{array}$ \\
\hline Summe (n/FxT) & 0,273 & 0,264 & 0,532 & 0,536 & 0,292 & 0,323 & 0,629 & 0,496 & 0,251 & 0,192 & 0,226 \\
\hline Individuenanzahl (n) & 191 & 148 & 383 & 363 & 164 & 228 & 445 & 278 & 181 & 128 & 127 \\
\hline Artenanzahl & 36 & 34 & 45 & 52 & 36 & 42 & 53 & 36 & 29 & 23 & 27 \\
\hline turnover & & 0,40 & & 0,39 & 0,32 & & 0,48 & 0,40 & & 0,41 & 0,45 \\
\hline $\begin{array}{l}\text { Summe Arten-/ } \\
\text { Individuenanzahlen } \\
\text { 1996: } 69 / 792 \\
\text { 1998: } 81 / 1127 \\
\text { 2000: } 67 / 718 \\
\end{array}$ & & & & & & & & & & & \\
\hline
\end{tabular}


1998 wurden auf allen Untersuchungsflächen 1127 Individuen mit insgesamt 81 Arten nachgewiesen (Tab. 1). Auf den Untersuchungsflächen 1 und 2 konnten jeweils 52 bzw. 53 Arten und auf der Kontrollfläche 3 konnten 23 Arten gefunden werden (Tab. 1). 1998 unterschieden sich die Aktivitätsdichten auf den Untersuchungsflächen 1 und 2 nicht, auf der Kontrollfläche 3 war die Aktivitätsdichte der Spinnen signifikant geringer (Chiquadrat Test, $\mathrm{p}<0,05)$. 36 Arten wurden 1998 auf der Untersuchungsfläche 0 mit einer Aktivitätsdichte von 0,273 Individuen pro Falle pro Tag gefangen (Tab. 1). Hier wurde Xysticus luctuosus gefunden, eine Art, die in Kategorie 2 der Roten Liste der Spinnentiere von Berlin und Brandenburg geführt wird (PLATEN et al. 1999). Die Art bevorzugt trockene Lebensräume, besiedelt die Streu von Laub- und Nadelwäldern und wurde zuletzt 1997 in einem Kiefernforst bei Bremsdorf (Kreis Oder-Spree) nachgewiesen (PLATEN et al. 1999).

Im Jahr 2000 wurden 718 Individuen mit 67 Arten auf den Untersuchungsflächen nachgewiesen. Auf den Untersuchungsflächen 1 und 2 wurden je 36 Arten, auf der Untersuchungsfläche 0 wurden 34 Arten und auf der Kontrollfläche 3 wurden 27 Arten gefunden (Tab. 1). Die Aktivitätsdichten blieben aufden Untersuchungsflächen 0 und 3 im Vergleich zu 1998 nahezu konstant und wiesen auf den Untersuchungsflächen 1 und 2 geringere Werte auf (Abb. 1). Die höchste Aktivitätsdichte der Spinnen wurde auch 2000 auf der Untersuchungsfläche 2 gefunden.

Insgesamt wurden in den Jahren 1996, 1998 und 2000105 Arten auf allen Untersuchungsflächen nachgewiesen. Die Artenaustauschvorgänge berechnet als turnover Raten der Spinnen zwischen 1996 und 1998 weisen auf der Untersuchungsfläche 2 einen höheren Wert auf $(0,48)$, als auf den Untersuchungsflächen $1(0,39)$ und $3(0,41)$. Zwischen 1998 und 2000 gab es kaum Unterschiede in den Artenaustauschraten zwischen den Untersuchungsflächen (Tab. 1).

Die Aktivitätsdichten der Spinnen auf der Kontrollfläche 3 wiesen zwischen 1996, 1998 und 2000 nur geringe Veränderungen auf (Abb. 1). Auf der Untersuchungsfläche 2 wurden 1998 deutlich höhere Werte der Aktivitätsdichten der Spinnen im Vergleich zu 1996 registriert (Abb. 1). Auf der Untersuchungsfläche 1 ergaben sich 1996 und 1998 hohe Aktivitätsdichten der Spinnen. Auf den Untersuchungsflächen 1 und 2 wurden im Jahr 2000 geringere Aktivitätsdichten gefunden als in den 


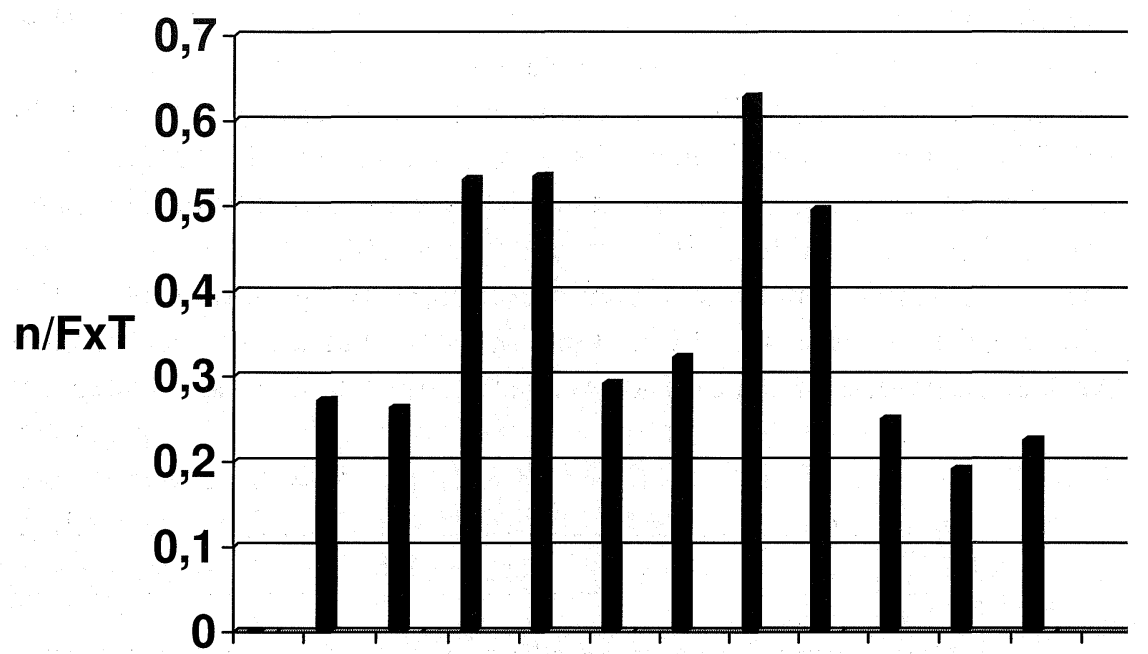

$\begin{array}{llllllllllllr}\text { Jahr } & 96 & 98 & 00 & 96 & 98 & 00 & 96 & 98 & 00 & 96 & 98 & 00 \\ \text { Fläche } & 0 & 0 & 0 & 1 & 1 & 1 & 2 & 2 & 2 & 3 & 3 & 3\end{array}$

Abb. 1: Aktivitätsdichten (Individuenanzahl pro Falle pro Tag $=\mathrm{n} / \mathrm{FxT}$ ) der Spinnen auf den Untersuchungsflächen (0-3) in den Jahren 1996, 1998 und 2000.

Fig. 1: The number of spiders collected per day per trap on the study areas (0-3) during the years 1996,1998 and 2000.

vorher untersuchten Jahren. Auf der Untersuchungsfläche 0 ergaben sich ähnlich geringe Aktivitätsdichten wie auf der Kontrollfläche 3, die Aktivitätsdichten blieben 1998 und 2000 annähernd konstant.

Bei einer Einteilung der nachgewiesenen Spinnenarten in eher xerophile Arten und in eher hygrophile Arten nach PLATEN et al. (1999) wird deutlich, dass die erhöhte Gesamtaktivitätsdichte auf der Untersuchungsfläche $2 \mathrm{im} \mathrm{Jahr} 1998$ vor allem aufhöheren Aktivitätsdichten von xerophilen Arten basiert (Abb. 2). Die Veränderungen der Aktivitätsdichten der hygrophilen Arten waren auf allen Untersuchungsflächen zwischen 1996 und 1998 um den Faktor 10 geringer. Von den xerophilen Arten auf der Unter- 
suchungsfläche 2 wies Trochosa terricola eine Veränderung ihrer Aktivitätsdichte zwischen 1996 und 1998 von + 90,9\% (+ 0,080 Individuen pro Falle pro Tag) auf, Euryopis flavomaculata eine Veränderung von $+84 \%$ $(+0,074$ Individuen pro Falle pro Tag), und Lepthyphantes flavipes wies eine Veränderung von $+23,5 \%(+0,016$ Individuen pro Falle pro Tag $)$ auf. Im Jahr 2000 wiesen die Aktivitätsdichten der xerophilen Arten auf den Untersuchungsflächen 1 und 2 erneut geringere Werte auf (Abb. 2), die jedoch höher waren als auf den Untersuchungsflächen 0 und 3.

Die Veränderungen der Gesamtaktivitätsdichten erklärt sich somit durch

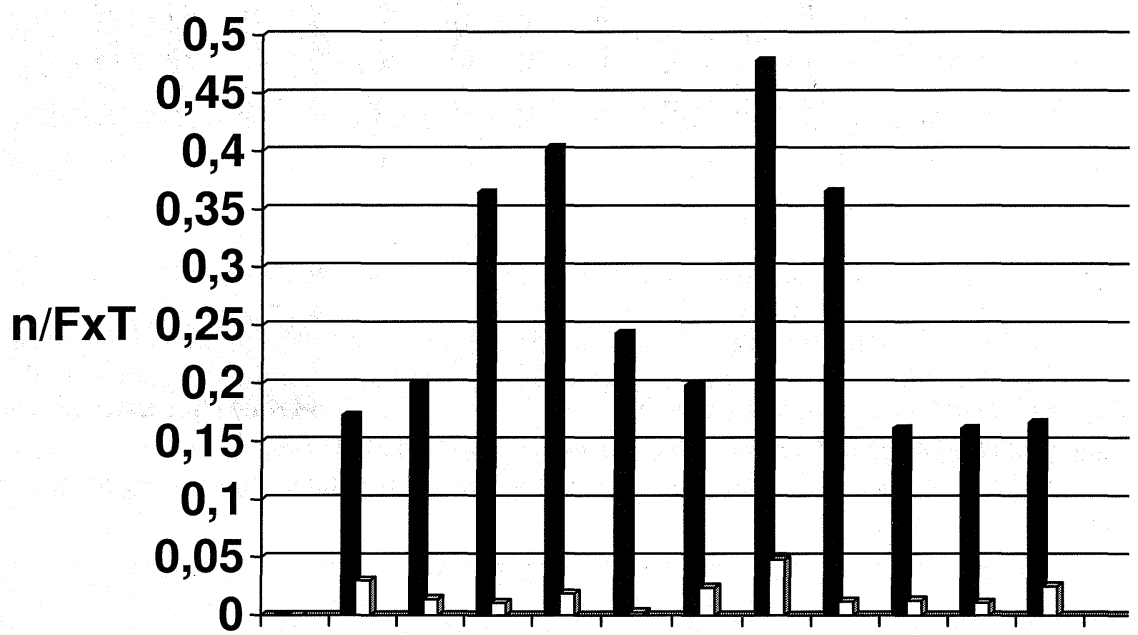

$\begin{array}{lllllllllllll}\text { Jahr } & 96 & 98 & 00 & 96 & 98 & 00 & 96 & 98 & 00 & 96 & 98 & 00 \\ \text { Fläche } & 0 & 0 & 0 & 1 & 1 & 1 & 2 & 2 & 2 & 3 & 3 & 3\end{array}$

Abb. 2: Aktivitätsdichten (Individuenanzahl pro Falle pro $\mathrm{Tag}=\mathrm{n} / \mathrm{FxT}$ ) der xerophilen $(\mathbf{\square})$ und hygrophilen ( $\square$ ) Spinnenarten auf den Untersuchungsflächen (0-3) in den Jahren 1996, 1998 und 2000.

Fig. 2: The numbers of the xerophilous ( $\square$ ) and the numbers of the hygrophilous ( $\square$ ) spider species collected per day per trap on the study areas (0-3) during the years 1996, 1998 and 2000 . 
die Veränderungen der Aktivitätsdichten der xerophilen Arten, während die Aktivitätsdichten der hygrophilen Arten weiter sehr gering blieben. So wiesen die Arten der Untersuchungsfläche 2, die im Vergleich von $1996 \mathrm{zu}$ 1998 besonders hohe Steigerungen ihrer Aktivitätsdichten aufwiesen, bei einem Vergleich von $1998 \mathrm{zu} 2000$ folgende Veränderungen auf: Trochosa terricola - 71,6 \% (- 0,063 Individuen pro Falle pro Tag), Euryopis flavomaculata - 21,6 \% (- 0,019 Individuen pro Falle pro Tag), Lepthyphantes flavipes - 36,7 \% (- 0,025 Individuen pro Falle pro Tag).

Die Spinnengemeinschaften auf den Untersuchungsflächen im Jahr 1996 wiesen hohe Übereinstimmungen hinsichtlich der reinen Artenübereinstimmungen (SÖRENSEN-Quotient) und auch hinsichtlich der Dominanzverhältnisse der gemeinsamen Arten (JACCARD-Index und WAINSTEIN-Index) auf den Untersuchungsflächen 2 und 3 auf (Tab. 2). Im Jahr 1998 wies die Gemeinschaft der Spinnen auf den behandelten Untersuchungsflächen 1 und 2 die höchsten Artenübereinstimmungen

Tab. 2: Artenübereinstimmungen (SÖRENSEN-Quotienten, JACCARD-Indices und WAINSTEIN-Indices in \%) der Spinnengemeinschaften auf den Untersuchungsflächen (03 ) in den Jahren 1996, 1998 und 2000.

Tab. 2: Correspondence of the spider communities (SÖRENSEN-, JACCARD- and WAINSTEIN-indices in \%) on the study areas (0-3) during the years 1996, 1998 and 2000.

\begin{tabular}{|c|c|c|c|c|c|c|c|c|c|c|c|c|c|}
\hline & & \multicolumn{4}{|c|}{ SÖRENSEN } & \multicolumn{4}{|c|}{ JACCARD } & \multicolumn{4}{|c|}{ WAINSTEIN } \\
\hline Fläche & & 0 & 1 & 2 & 3 & 0 & 1 & 2 & 3 & 0 & 1 & 2 & 3 \\
\hline Jahr & & & & & & & & & & & & & \\
\hline 1996 & 0 & - & - & & - & - & - & - & - & 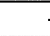 & - & - & - \\
\hline & 1 & & & 52,8 & 48,6 & & - & 35,9 & 32,1 & & - & 15,2 & 13,8 \\
\hline & 2 & & & & 59,1 & & & - & 42,0 & & & - & 27,5 \\
\hline & 3 & & & & & & & & & & & & - \\
\hline 1998 & 0 & - & 57,7 & 48,8 & 43,3 & - & 40,6 & 32,3 & 27,6 & . & 5,3 & 5,6 & 2,1 \\
\hline & 1 & & - & 69,5 & 42,8 & & - & 53,3 & 27,3 & & - & 28,0 & 3,7 \\
\hline & 2 & & & & 42,3 & : & & - & 26,8 & & & & 11,7 \\
\hline & 3 & & & & & & & & 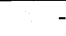 & & & & - \\
\hline 2000 & 0 & & 60,0 & 45,7 & 45,9 & - & 42,8 & 29,6 & 29,8 & 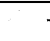 & 23,5 & 14,3 & 8,9 \\
\hline & 1 & & - & 58,3 & 50,8 & 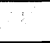 & - & 41,2 & 34,0 & & S & 15,5 & 8,8 \\
\hline & 2 & & & & 76,2 & & & - & 61,5 & & & - & 27,4 \\
\hline & 3 & & & & - & & & & - & & & & - \\
\hline
\end{tabular}


(SÖRENSEN-Quotient) auf, und bei Berücksichtigung der Dominanzverhältnisse in Form der Aktivitätsdichten (JACCARD-Index und WAINSTEIN-Index) zeigten sich höhere Übereinstimmungen auf den Untersuchungsflächen 1 und 2, während insbesondere die Spinnengemeinschaften zwischen den Untersuchungsflächen 1 und 3 verschieden waren (Tab. 2). Wie die sehr geringen WAINSTEIN-Indices belegen, wurde auf der Untersuchungsfläche 0 im Jahr 1998 eine völlig andere Spinnengemeinschaft angetroffen, als auf allen anderen Untersuchungsflächen (Tab. 2). Hier dominierte die Artengemeinschaft von Euryopis flavomaculata, Lepthyphantes flavipes, Robertus lividus, Trochosa terricola, Walckenaeria furcillata und Zora spinimana. Im Jahr 2000 wies die Gemeinschaft der Spinnen hohe Werte der Artenübereinstimmungen (SÖRENSEN-Quotient) zwischen den Untersuchungsflächen auf (Tab. 2). Die geringen WAINSTEIN-Indices zwischen den Untersuchungsflächen 0 und 3 sowie 1 und 3 deuten an, dass sich die Aktivitätsdichten der dominanten Spinnenarten auf diesen Untersuchungsflächen deutlich unterschieden.

Werden zur Beschreibung der Veränderungen der Artengemeinschaften der Spinnen nur diejenigen Arten betrachtet, welche pro Untersuchungsfläche und pro Jahr mehr als $5 \%$ des jeweiligen Gesamtfanges pro Untersuchungsfläche erreichen (dominante Arten), so waren im Jahr 1996 auf den Untersuchungsflächen 1 und 3 acht Arten, auf der Untersuchungsfläche 2 sechs Arten vorhanden, welche insgesamt 54,2\% bis $69,8 \%$ des Gesamtfanges ausmachten (Tab. 3). Besonders hohe Anteile am Gesamtfang erreichten Lepthyphantes flavipes auf den Untersuchungsflächen 2 und 3 sowie Walckenaeria atrotibialis auf allen Untersuchungsflächen. Im Jahr 1998 war die Anzahl der Arten geringer, die mehr als $5 \%$ des Gesamtfanges pro Untersuchungsfläche erreichten. Die Artenaustauschraten der dominanten Arten zwischen den Jahren 1996 und 1998 waren hoch, z.B. waren auf der Untersuchungsflächen 2 mehr als $80 \%$ der dominanten Arten verändert. Die Artenaustauschraten der dominanten Arten zwischen 1998 und 2000 wiesen erheblich geringere Werte auf.

Wenn die dominanten Spinnenarten für eine Berechnung der Artenübereinstimmung berücksichtigt werden (SÖRENSEN-Quotient der dominanten Arten), so wird deutlich, dass im Jahr 1996 die höchsten Übereinstimmungen der dominanten Artengemeinschaften auf den 
Tab. 3: Prozentuale Anteile der dominanten Spinnenarten ( $>5 \%$ ) am Gesamtfang auf den Untersuchungsflächen (0-3) im Jahr 1996, 1998 und 2000, Summen, Anzahl der dominanten Arten pro Untersuchungsfläche und turnover der dominanten Spinnenarten.

Tab. 3: The dominant spider species ( $>5 \%)$ on the study areas (0-3) during the years 1996, 1998 and 2000, sum, numbers of the dominant species per study area, and turnover of the dominant species per year.

\begin{tabular}{|c|c|c|c|c|c|c|c|c|c|c|c|}
\hline Fläche & 0 & 0 & 1 & 1 & 1 & 2 & 2 & 2 & 3 & 3 & 3 \\
\hline Jahr & 1998 & 2000 & 1996 & 1998 & 2000 & 1996 & 1998 & 2000 & 1996 & 1998 & 2000 \\
\hline 1. Agroeca brunnea & & & & & & 6,6 & & & 8,8 & & 7,0 \\
\hline 2. Agroeca proxima & & & & & & & & & & 7,5 & \\
\hline $\begin{array}{l}\text { 3. Centromerus } \\
\text { incilium }\end{array}$ & & & 5,2 & & & & & & & & \\
\hline $\begin{array}{l}\text { 4. Centromerus } \\
\text { sylvaticus }\end{array}$ & & & & & & & & & & & 5,5 \\
\hline $\begin{array}{l}\text { 5. Euryopis } \\
\text { flavomaculata }\end{array}$ & 6,3 & 18,9 & 5,5 & 11,5 & 21,3 & & 14,1 & 14,0 & & & \\
\hline $\begin{array}{l}\text { 6. Lepthyphantes } \\
\text { flavipes }\end{array}$ & 10,9 & & & & & 16,2 & 10,8 & 8,6 & 17,6 & 28,7 & 32,3 \\
\hline 7. Macrargus rufipes & & & & & & & & & 5,5 & & \\
\hline $\begin{array}{l}\text { 8. Pelecopsis } \\
\text { radicicola }\end{array}$ & & & & & & 8,3 & & 24,5 & & & \\
\hline $\begin{array}{l}\text { 9. Pocadicnemis } \\
\text { pumila }\end{array}$ & & & & 5,2 & & & & & & & \\
\hline $\begin{array}{l}\text { 10. Phrurolithus } \\
\text { festivus }\end{array}$ & & & 6,8 & & & & & & & & \\
\hline 11. Robertus lividus & 5,2 & & & & & & & & & & \\
\hline $\begin{array}{l}\text { 12. Trochosa } \\
\text { terricola }\end{array}$ & 6,3 & & 13,0 & & 10,9 & & 14,1 & 5,0 & & 29,5 & 6,3 \\
\hline
\end{tabular}




\begin{tabular}{|c|c|c|c|c|c|c|c|c|c|c|c|}
\hline Fläche & 0 & 0 & 1 & 1 & 1 & 2 & 2 & 2 & 3 & 3 & 3 \\
\hline Jahr & 1998 & 2000 & 1996 & 1998 & 2000 & 1996 & 1998 & 2000 & 1996 & 1998 & 2000 \\
\hline $\begin{array}{l}\text { 13. Walckenaeria } \\
\text { atrotibialis }\end{array}$ & & & 11,2 & & & 12,2 & & & 11,0 & & \\
\hline $\begin{array}{l}\text { 14. Walckenaeria } \\
\text { cucullata }\end{array}$ & & & & & & 5,2 & & & 8,3 & & \\
\hline $\begin{array}{l}\text { 15. Walckenaeria } \\
\text { dysderoides }\end{array}$ & & & 7,3 & & & 5,7 & & & 6,0 & & \\
\hline $\begin{array}{l}\text { 16. Walckenaeria } \\
\text { furcillata }\end{array}$ & 7,8 & 11,5 & & & & & & 5,4 & 6,0 & & \\
\hline $\begin{array}{l}\text { 17. Xerolycosa } \\
\text { nemoralis } \\
\end{array}$ & & & & 11,2 & & & & & & & \\
\hline 18. Zelotes clivicola & & 12,1 & 8,8 & 9,9 & 18,3 & & & & & & \\
\hline \begin{tabular}{|l|}
19. Zelotes \\
subterraneus
\end{tabular} & & 9,4 & 7,8 & 8,2 & 7,9 & & 8,8 & 7,2 & & & 7,0 \\
\hline 20. Zora spinimana & 14,6 & & & & & & 5,9 & 5,4 & 6,6 & & \\
\hline Summe $(\%)$ & 51,1 & 51,9 & 65,6 & 46,0 & 58,4 & 54,2 & 53,7 & 70,1 & 69,8 & 65,7 & 58,1 \\
\hline Artenanzahl & 6 & 4 & 8 & 5 & 4 & 6 & 5 & 7 & 8 & 3 & 5 \\
\hline turnover & & 0,60 & & 0,53 & 0,33 & & 0,81 & 0,16 & & 0,63 & 0,50 \\
\hline
\end{tabular}


Untersuchungsflächen 2 und 3 gefunden wurden (Tab. 4). Im Jahr 1998 fällt auf, dass es keine übereinstimmende dominant vorkommende Spinnenart zwischen den Untersuchungsflächen 1 und 3 gab (Tab. 4). Im Jahr 2000 wiesen die dominanten Spinnenarten zwischen den Untersuchungsflächen 0 und 1 vergleichsweise hohe Werte auf, geringere Übereinstimmungen wurden zwischen der Kontrollfläche 3 und den übrigen Untersuchungsflächen berechnet (Tab. 4).

Tab. 4: Artenübereinstimmungen (SÖRENSEN-Quotienten) der dominanten Spinnenarten (>5\%) auf den Untersuchungsflächen (0-3) in den Jahren 1996, 1998 und 2000.

Tab. 4: Correspondence (SÖRENSEN-index) of the dominant spider species $(>5 \%)$ on the study areas (0-3) during the years 1996, 1998 and 2000.

\begin{tabular}{|l|r|r|r|r|r|}
\hline & & \multicolumn{4}{|c|}{ SÖRENSEN } \\
\hline Fläche & & 0 & 1 & 2 & \multicolumn{1}{|c|}{3} \\
\hline Jahr & & & & & \\
\hline 1996 & 0 & & & & \\
\hline & 1 & & & 28,6 & 25,0 \\
\hline & 2 & & & - & 71,4 \\
\hline & 3 & & & & \\
\hline 1998 & 0 & & 9,0 & 72,7 & 44,4 \\
\hline & 1 & & & 40,0 & 0 \\
\hline & 2 & & & & 50,0 \\
\hline & 3 & & & & \\
\hline 2000 & 0 & & 37,5 & 27,3 & 11,1 \\
\hline & 1 & & - & 27,3 & 22,2 \\
\hline & 2 & & & & 25,0 \\
\hline & 3 & & & & - \\
\hline
\end{tabular}




\section{DISKUSSION}

Mit der angewandten Methode werden nur bodenbesiedelnde Spinnen erfasst. Die Vor- und Nachteile der Methode sind ausführlich dargestellt und diskutiert worden (SUNDERLAND et al. 1995, POWELL et al. 1996, MC EWEN 1997). Weitere Spinnenarten aus spezifischen Habitaten wie etwa der Kronenregion (SIMON 1995) oder aus dem Stammbereich (NICOLAI 1986, 1995) können mit der hier benutzten Methode nicht nachgewiesen werden.

Aus Berlin und Brandenburg sind bisher 641 Spinnenarten bekannt (PLATEN et al. 1999). Somit wurden 16,4 \% aller Arten aus Berlin und Brandenburg in den hier untersuchten Kiefernforsten (Summe 105 Arten) nachgewiesen. Von 114 Arten, die nach PLATEN et al. (1999) auch aus Kiefernforsten von Berlin und Brandenburg gemeldet sind, wurden in dieser Studie 66 Arten gefunden (=57,9\%). Die nachgewiesene Artenanzahl der Spinnen ist damit vergleichsweise hoch. ALBERT (1976) weist mit mehreren verschiedenen Methoden in einem Buchenwald insgesamt 85 Spinnenarten nach, von denen 20 Arten in Bodenfallen gefunden wurden, die den hier eingesetzten Fallen entsprechen. UHLENHAUT et al. (1987) weisen in einem lichten Eichenwald 75 Spinnenarten und in einem Fichtenforst 40 bodenbesiedelnde Spinnenarten nach. PLATEN \& KOWARIK (1995) finden 63 bodenbesiedelnde Spinnenarten in einem Robinienaltbestand in Berlin, davon etwa $50 \%$ von Spinnen mit einem Schwerpunktvorkommen in Wäldern. Auf den hier untersuchten Flächen der Kiefernforste, auf denen Umbaumaßnahmen durchgeführt worden waren, wurden in allen Untersuchungsjahren höhere Artenanzahlen gefunden als auf der unbehandelten Kontrollfläche. Auf allen Untersuchungsflächen wurden hohe Artenaustauschraten von einer Vegetationsperiode zur übernächsten Vegetationsperiode gemessen. Die Artengemeinschaften pro Untersuchungsfläche verändern sich daher stark. Dementsprechend sind Untersuchungen in einer Vegetationsperiode nicht geeignet, das Artenrepertoire der Spinnen eines Kiefernforstes darzustellen. Die hohen Artenaustauschraten wurden sowohl für die Gesamtheit der Spinnenarten pro Untersuchungsfläche als auch für die dominanten Spinnenarten festgestellt. Zwar waren in den verschiedenen Untersuchungsjahren unterschiedliche Arten auf den Untersuchungsflächen präsent, die Áktivitätsdichten insgesamt unterschieden sich auf den Untersuchungsflächen 1 und 
3 in den Untersuchungsjahren 1996 und 1998 jedoch nicht signifikant. Auf der Untersuchungsfläche 2 kam es zu einer Erhöhung der Gesamtaktivitätsdichte vor allem von xerophilen Arten. Nach Durchführung der Umbaumaßnahmen herrschten am Boden auf der Untersuchungsfläche 1 höhere Bodentemperaturen und auch höherer Lichteinfall als auf der Kontrollfläche 3, wo geringere Bodentemperaturen und geringere Lichtintensitäten gemessen wurden (NICOLAI 2000), und damit ergeben sich auch höhere relative Luftfeuchten der bodennahen Luftschicht (STOUTJESDIJK \& BARKMAN 1992). Im Jahr 2000 waren die Aktivitätsdichten auf den Untersuchungsflächen 0 und 3 nahezu unverändert, während auf den Untersuchungsflächen 1 und 2 geringere Aktivitätsdichten vorkamen, die jedoch höhere Werte aufwiesen, als auf den Untersuchungsflächen 0 und 3. Die geringeren Aktivitätsdichten auf den Untersuchungsflächen 1 und $2 \mathrm{im}$ Jahr 2000 betrafen vor allem xerophile Arten, die auf den Untersuchungsflächen 1998 in hohen Aktivitätsdichten gefunden worden waren.

Werden nach der Methode von SCHEURIG et al. (1996) zur Beschreibung des typischen Artenrepertoires eines Habitats die dominanten Arten betrachtet, die auf allen Untersuchungsflächen gleichzeitig mehr als $5 \%$ des jeweiligen Gesamtfanges erreichen, können für 1996 nur die Arten Walckenaeria atrotibialis und $W$. dysderoides als typische Besiedler des Bodenstratums der Kiefernforste benannt werden. 1998 und 2000 tritt keine Art gleichzeitig auf allen Untersuchungsflächen dominant auf. Die Artengemeinschaften der dominanten Arten scheinen sich also auf den Untersuchungsflächen unabhängig voneinander weiter zu entwickeln, wofür auch die relativ geringen Artenaustauschvorgänge zwischen 1998 und 2000 auf den Untersuchungsflächen 1 und 2 sprechen.

Von den gefundenen dominanten Arten sind Agroeca proxima, Xerolycosa nemoralis, und Zelotes clivicola als mäßig häufig (16 - 100 aktuelle Vorkommen), Pocadicnemis pumila und Walckenaeria furcillata als häufig (> 100 aktuelle Vorkommen) und alle anderen als sehr häufig (aktuell nahezu überall nachgewiesen) in Berlin und Brandenburg eingestuft worden (PLATEN et al. 1999).

In Mischwäldern wurde eine starke Heterogenität des Artenbesatzes pro Fläche für phytophage Käfer gefunden (FOTOPOULOS \& NICOLAI 2002). Ob die Heterogenität des Artenbesatzes der Spinnen pro Fläche in 
Kiefernforsten Brandenburgs generell zutrifft, und ob die Artengemeinschaften auf den unterschiedlich behandelten Untersuchungsflächen weiter divergieren, können Themen weiterer Untersuchungen sein.

\section{ZUSAMMENFASSUNG}

Die bodenbesiedelnden Spinnen von vier aneinander angrenzenden Kiefernforsten in Brandenburg wurden 1996, 1998 und 2000 mit Bodenfallen erfasst. Drei der Kiefernforste waren 1995 unterschiedlich vorbehandelt worden, eine unbehandelte Untersuchungsfläche diente der Kontrolle. Insgesamt wurden 105 verschiedene Spinnenarten auf den Untersuchungsflächen nachgewiesen, wobei auf der Kontrollfläche in allen Jahren die geringsten Artenanzahlen und Aktivitätsdichten pro Untersuchungsfläche gefunden wurden. Durch die Vorbehandlungen der Kiefernforste kam es zu Auflichtungen, wodurch in erhöhtem Maße xerophile Arten die Untersuchungsflächen besiedeln konnten. Insgesamt waren die Spinnengemeinschaften der Untersuchungsflächen sehr heterogen, wiesen starke Artenaustauschvorgänge von Jahr zu Jahr auch für dominante Arten auf und reflektierten die ablaufenden Veränderungen in den Kiefernforsten. Auch Arten, die für das Land Brandenburg als selten gemeldet worden sind, konnten in den Kiefernforsten nachgewiesen werden.

Dank: Wir danken dem Landschaftsfördervein Nuthe-Nieplitz-Niederung für die Möglichkeiten der Freilandarbeiten, für vielfältige Unterstützungen und für die Durchführung der Umbaumaßnahmen. Einem anonymen Gutachter danken wir für konstruktive Verbesserungsvorschläge zu dem Manuskript.

\section{LITERATUR}

ALBERT R. (1976): Zusammensetzung und Vertikalverteilung der Spinnenfauna in Buchenwäldern des Solling. - Faun. ökol. Mitt. 5: 65-80

AMMER U. \& K. KÜBLER (1998): Forschung für eine zukunftsorientierte Waldwirtschaft. Ermittlung des Forschungsbedarfs in der BRD im Auftrag des BMBF, Bonn. Lehrstuhl für Landnutzungsplanung und Naturschutz. Freising. $175 \mathrm{~S}$.

BUNDESAMT FÜR NATURSCHUTZ (Hrsg.) (1999): Daten zur Natur 1999. BfN Landwirtschaftsverlag, Münster. $226 \mathrm{~S}$. 
ELLENBERG H. (1996): Vegetation Mitteleuropas mit den Alpen. Ulmer, Stuttgart. $1095 \mathrm{~S}$.

FOTOPOULOS L. \& V. NICOLAI (2002): Vergleiche der Rüsselkäferfauna (Col., Curculionoidea) an zwei einheimischen und zwei fremdländischen Baumarten. - Neobiota 1: $181-190$

HEIMER S. \& W. NENTWIG (1991): Spinnen Mitteleuropas. Parey, Hamburg. 490 S.

MC EWEN P. (1997): Sampling, handling and rearing insects. In: D.R. DENT \& M.P. WALTON (eds.): Methods in ecological and agricultural entomology. CAB International, Oxon, U.K.: 5-26

NICOLAI V. (1986): The bark of trees: thermal properties, microclimate and fauna.

- Oecologia 69: 148-160

NICOLAI V. (1995): The ecological significances of trees bark during ecosystem dynamics. - Spixiana 18: 187-199

NICOLAI V. (2000): Ökologische Untersuchungen während Umbauprozessen von Kiefernforsten in Mischwälder. Schriftenreihe Agraria 29: 1-100, Kovac Verlag, Hamburg.

NICOLAI V. \& D. MACHANDER (2000): Kieferntotholz als Überwinterungshabitat für Arthropoden nebst Erstmeldung von Leptusa norvegica (Col., Staphylinidae) für Brandenburg. - Entomol. Nachr. Ber. 44: 171-174

PETERKEN G.F. (1996): Natural woodland. Cambridge University Press, Cambridge. $522 \mathrm{~S}$.

PLATEN R., \& I. KOWARIK (1995): Dynamik von Pflanzen-, Spinnen-, und Laufkäfergemeinschaften bei der Sukzession von Trockenrasen zu Gehölzgesellschaften auf innerstädtischen Bahnbrachen in Berlin. - Verh. Ges. Ökol. 24: 431-439

PLATEN R., B. v. BROEN, A. HERRMANN, U.M. RATSCHKER \& P. SACHER (1999): Gesamtartenliste und Rote Liste der Webspinnen, Weberknechte und Pseudoskorpione des Landes Brandenburg (Arachnida: Araneae, Opiliones, Pseudoscorpiones) mit Angaben zur Häufigkeit und Ökologie. - Naturschutz Landschaftspf. Brandenburg, Beilage zu Heft 8 (2): 1-79

PLATNICK N.I. (1997): Advances in Spider Taxonomy 1992-1995 with Redescriptions 1940-1980. New York Entomological Society, New York, 976 S.

POTT R. (1997): Von der Urlandschaft zur Kulturlandschaft - Entwicklung und Gestaltung mitteleuropäischer Kulturlandschaften durch den Menschen. - Verh. Ges. Ökol. 27: 5-26 POWELL W., M.P. WALTON \& M.A. JERVIS (1996): Populations and Communities. In: M.A. JERVIS \& N.A.C. KIDD (eds.): Insect Natural Enemies. Chapman \& Hall, London: 223-292

REMMERT H. (1998): Spezielle Ökologie Terrestrische Systeme. Springer, Berlin, Heidelberg, New York. $257 \mathrm{~S}$.

ROBERTS M.J. (1995): Spiders of Britain and Northern Europe. Collin Field Guide, Harper Collins, London. $383 \mathrm{~S}$.

SCHERZINGER W. (1996): Naturschutz im Wald. Qualitätsziele einer dynamischen Waldentwicklung. Ulmer, Stuttgart. $447 \mathrm{~S}$.

SCHEURIG M., W. HOHNER, D. WEICK, F. BRECHTEL \& L. BECK (1996): Laufkäferzönosen südwestdeutscher Wälder - Charakterisierung, Beurteilung und Bewertung von Standorten. - Carolinea 54: 91-138 
SEELEMANN S., G.SEELEMANN \& H.SICHTING (1996): Errichtung und Sicherung schutzwürdiger Teile von Natur und Landschaft mit gesamtstaatlich repräsentativer Bedeutung. Projekt: Nuthe-Nieplitz-Niederung, Brandenburg. - Natur und Landschaft 71: $318-323$

SIMON U. (1995): Untersuchung der Stratozönosen von Spinnen und Weberknechten (Arachn.: Araneae, Opilionida) an der Waldkiefer (Pinus sylvestris L.). Dissertation, Technische Universität Berlin, Fachbereich Umwelt und Gesellschaft. Wissenschaft und Technik Verlag, Berlin. $142 \mathrm{~S}$.

STOUTJESDIJK P. \& J.J. BARKMAN (1992): Microclimate, vegetation and fauna. Opulus Press, Schweden. $216 \mathrm{~S}$.

SUNDERLAND K.D., G.R. DE SNOO, A. DINTER, T. HANCE, J. HELENIUS, P. EPSON, B. KROMP, F. SAMU, N.W. SOUTHERTON, S. TOFT \& B. ULBER (1995): Density estimation for invertebrate predators in agroecosystems. - Acta Jutlandica 70: $134-164$

UHLENHAUT H., V. NICOLAI \& W. NENTWIG (1987): Die Spinnenfauna der Lahnberge bei Marburg. - Decheniana 140: 59-65

WIEHLE H. (1956): Linyphiidae - Baldachinspinnen. In: Die Tierwelt Deutschlands und der angrenzenden Meeresteile. 44. Teil, G. Fischer, Jena. $337 \mathrm{~S}$.

WIEHLE H. (1960): Micryphantidae - Zwergspinnen. In: Die Tierwelt Deutschlands und der angrenzenden Meeresteile. 47. Teil, G. Fischer, Jena. $620 \mathrm{~S}$.

Prof. Dr. Volker NICOLAI, Technische Universität Berlin, Fakultät VII, Institut für Ökologie, Fachgebiet Zoologie, FR 1-1, Franklinstr. 28/29, D-10587 Berlin, e-mail: nicolai.v.@web.de Andreas HERRMANN, Landesumweltamt Brandenburg, Postfach 601061, D-14410 Potsdam, e-mail: Andreas.Herrmann@lua.brandenburg.de 